\title{
FILOSOFÍA COGNITIVA DE LA LÓGICA Y ANTI-PSICOLOGISMO
}

\author{
Alejandro Ramírez F. \\ Universidad de Chile \\ alramire@uchile.cl
}

\begin{abstract}
RESUMEN / ABSTRACT
La discusión sobre el psicologismo lógico, que parecía ya superada desde Frege-Husserl, ha vuelto a posicionarse hoy en la filosofía de la lógica. Se examina, en primer lugar, la idea según la cual lo que hoy se entiende por lógica respondería en lo esencial a las tesis defendidas por el anti-psicologismo. En segundo término, se plantea que algunos enfoques actuales en ciencia cognitiva, como el de la facultad cognitiva proto-lógica de R.Hanna, y el de los dominios cognitivos, de Stennings y van Lambalgen, permiten apoyar la idea de que la naturaleza de la lógica va más allá de los atributos antipsicologistas.
\end{abstract}

PALABRAS CLAVE: lógica, anti-psicologismo, protológica, filosofía, cognición.

\section{Cognitive Philosophy of Logic and the Anti-PSychologism}

Discussion on logic psychologism, which seemed to be over since Frege-Husserl, has now positioned itself in philosophy of logic. In the first place, the idea according to which what is currently understood as logics would essentially respond to thesis defended by anti-psychologism is analyzed. In the second place, it is set forth that some cognitive science current approaches, such as R. Hanna protologic cognitive ability and Stennings and van Lambalgen cognitive domains, support the idea that the nature of logic goes beyond these anti-psychologist attributes.

KEYWORDS: Logic, Anti-psychologism, protologic, philosophy, cognition. 
La naturaleza de la lógica es significativamente revelada por la psicología cognitiva.

R. Hanna ${ }^{1}$

Los argumentos de Frege en contra del psicologismo sospecho que son menos concluyentes, por lo menos para alguna forma de psicologismo más plausible, que lo que hoy en día se puede suponer

Haack $^{2}$

\section{Introducción}

RA ¿Apoya o refuta la ciencia cognitiva al psicologismo lógico? ¿Significan los estudios cognitivos sobre el rol de la lógica en el razonamiento humano un retorno al psicologismo de Beneke (1798-1854), de Sigwart (1830-1940) o de W. Wundt, doctrina que parecía ya superada por Frege y Husserl? M. Dummett, refiriéndose justamente a la supuesta falta de decisión de Frege y Husserl para distinguir más claramente entre lógica y psicología, advierte del peligro del supuesto resurgimiento del psicologismo, del cual la ciencia cognitiva habría hoy tomado la bandera de lucha. En consecuencia, dice Dummett: "Las estrategias de defensa empleadas por Husserl y Frege no servirán demasiado más: los invasores pueden ser repelidos solamente corrigiendo las fallas de las teorías positivas de aquellos dos pioneros" (citado en Jacquette 2003, p. 7).

El problema del psicologismo, el invasor, según Dummett, ha sido uno de los temas centrales de la filosofía de la lógica contemporánea. Su relevancia radica en que la naturaleza de la lógica ha quedado determinada hasta hoy, a partir de Bolzano, Herbart, Frege y Husserl, como una disciplina definida antipsicológicamente, esto es, como una teoría estrictamente formal, independiente del tópico, a priori, cuyos objetos son relaciones entre enunciados y cuya tarea es determinar la validez de las estructuras o esquemas de los argumentos o de los razonamientos (en términos cognitivos), sin alusión alguna a contenidos, contextos, dominios, subjetividad, facultades o procesos mentales. La simbolización es, justamente, el vehículo de esa imagen objetiva de la lógica. Como afirma G. Priest (citado en Hjortland 2017, p. 63): "La noción central de la lógica es la validez, y su comportamiento es el principal asunto de las teorías lógicas. Dar una aproximación de la validez requiere dar cuenta de otras nociones, tales como la negación y el condicional".

1 Véase, sobre la concepción cognitiva de la lógica y el anti-psicologismo, R. Hanna 2006, p. xix.

2 S. Haack, 1991, p. 263, en su análisis de las lógicas divergentes. 
Los diversos estudios en ciencia cognitiva, en especial en psicología del razonamiento y en inteligencia artificial, han explorado y establecido las relaciones entre lógica y razonamiento tal como éste último se da en términos reales, en situaciones fácticas, sea en la ciencia o en la vida cotidiana ${ }^{3}$. Ello, a primera vista, daría un apoyo a la tesis de Dummett sobre una invasión neo-psicologista en lógica; sin embargo, argumentaremos que:

(a) la ciencia cognitiva hoy no es un retorno al psicologismo, y que

(b) ello tampoco implica que la naturaleza de la lógica deba entenderse estrictamente en los términos expuestos por el anti-psicologismo, como lo entendieron Frege y Husserl.

El ámbito en el cual ubicamos esta discusión es el de la relación filosofía de la lógica y cognición, por cuanto el psicologismo y anti-psicologismo son aproximaciones filosóficas a la esencia de la lógica. En ciencia cognitiva la relación lógica-cognición se la ha entendido, en la mayoría de los casos, en la dirección de izquierda a derecha, esto es, mediante la pregunta: cuál es el rol específico que cumple la idea de la lógica (formal simbólica) como conjunto de reglas válidas de inferencia en un agente cognitivo que debe enfrentarse a un cierto problema y que intenta resolver mediante un argumento. Las respuestas han sido variadas: van desde las tesis como las de A. Goldman (1986) que no ven ningún rol determinante, a otras, como las de la Inteligencia artificial y la abducción (Carnota 1995, Magnani 2001, Kowalski 2010, Aliseda 1998, 2006), que sí plantean roles más o menos claros y determinantes.

Sin embargo, la perspectiva que se adopta aquí es la inversa, la cual ha recibido muy poca atención en la filosofía de la lógica. Dicha mirada inversa a la mirada clásica va, ahora, de derecha a izquierda, esto es intenta examinar no el rol e incidencia de la lógica en la ciencia cognitiva sino que se propone determinar la influencia de la ciencia cognitiva en cuál sea la naturaleza de la lógica. Cabe mencionar que también hay posturas que cabría denominar bidireccionales, como por ejemplo la de McNamara (1994), quien defiende que tanto la lógica, como tema de la filosofía de la lógica, como la psicología cognitiva pueden y deben influirse y beneficiarse mutuamente.

La perspectiva que interesa aquí es la que conduce a interrogarse si la ciencia cognitiva puede influir en la formulación de la naturaleza de la lógica: qué hace la lógica realmente, con qué lo hace, por qué, con qué fin. El problema de qué sea la lógica

3 Las teorías cognitivas acerca del razonamiento son hoy muy variadas. Los principales enfoques vigentes y en discusión son las teorías de la lógica mental, cuyo antecedente fue el pensamiento de Piaget (Rips 2008), la tesis de los razonamientos basados en modelos mentales (Johnson Laird 1991, 1995, 1998 2005, Tversky B. 2005), la no monotonía y la inteligencia artificial (Kowalski 2010), las teorías heurísticas del razonamiento (Fernández y Carretero 1995), los enfoques duales (V. Thomson 2010, Verschueren y Schaeken 2010) y en general las actuales teorías integrativas. 
parece que se mantiene hoy tal como lo dejara Mill: "Encontramos tanta diversidad en la definición de la lógica como en la manera de tratar sus detalles” (J. S. Mill 1917, p. 9).

La pregunta por la influencia de la ciencia cognitiva en la reflexión sobre la naturaleza de la lógica, lo que, en otros términos, equivale a una idea de una filosofía cognitiva de la lógica, posee al menos estos dos fundamentos: 1) la analogía que se puede establecer con la actual filosofía cognitiva de la ciencia, en la que la ciencia cognitiva ha introducido nuevas perspectivas acerca de los problemas clásicos de esa disciplina, como son los planteamientos de Giere $(2002,2004)$ y la ciencia como cognición distribuida, N. Nersessian (2008) y los razonamientos basados en modelos o P. Thagard (1993) y el enfoque computacional de las teorías científicas; 2) la preocupación actual de varios autores sobre el carácter cognitivo último de la lógica, por ejemplo Hanna, Goldman, Stennings y van Lambalgen, Stelzer, cuyas ideas serán examinadas en lo que sigue.

En este contexto general, el tema del psicologismo-anti-psicologismo lógico tiene un lugar central del que nos ocuparemos aquí específicamente ${ }^{4}$. Se plantea la siguiente tesis de filosofía de la lógica:

Los desarrollos actuales en ciencia cognitiva no determinan, pero sí dan un apoyo a la idea de que la naturaleza de la lógica no queda determinada por la dicotomía psicologismo / anti-psicologismo, ni radicaría en última instancia en la idea de forma. Se examinan dos de dichos desarrollos: los dominios cognitivos (Stennings y van Lambalgen) y las facultades cognitivas (Hanna). El cognitivismo permitiría aunar ambas dimensiones, filosofía de la lógica y cognición, lo que significa que la naturaleza de lo lógico debe entenderse como un asunto de mayor complejidad.

No debe dejarse de lado, con todo, que el anti-psicologismo siempre reaparece una y otra vez en la filosofía de la lógica y con razones no débiles. Por ejemplo, L. Rips (1994, p.10) expone lo que podría ser una postura actual del antipsicologista: que no debemos confundir el "Razonamiento deductivo" con la "Inferencia deductiva"; el primero se refiere a una relación entre enunciados, que no tiene conexión alguna con estados psicológicos, en tanto la segunda tiene que ver con relaciones entre procesos de transformación de información; que lo primero tiene que ver con la disciplina lógica en tanto que lo segundo con capacidades humanas fácticas. Pero, centrarse en distinciones como ésta, bastante comunes actualmente, no obstante ser completamente ciertas en sí mismas, impiden replantearse el problema del antipsicologista a la luz de la ciencia cognitiva, como lo intentamos hacer en este artículo.

Cabe observar, finalmente, que no se trata de una determinación de la cognición en la lógica misma en cuanto métodos de validez, procedimientos de deducción, prueba, etc., cuestión que sí sería anti-psicologismo, esto es reducir las leyes lógicas a leyes psicológicas o cognitivas; se trata de una relación entre cognición y su posible influencia en hacer entender la naturaleza de la lógica.

$4 \quad$ El anti-psicologismo lógico, que se lo puede rastrear hasta Kant (2000), no ha sido una teoría filosófica entre otras; ha sido determinante en lo que hoy se entiende por lógica. 
En la sección 2 siguiente se examina la idea según la cual el anti-psicologismo ha sido una teoría dominante en la filosofía de la lógica. En la sección 3 se analizan dos aproximaciones que, proponemos, dan apoyo a la tesis expresada anteriormente, que son: el enfoque de los dominios cognitivos (Stennings y van Lambalgen) y la idea de facultad protológica (Hanna). La conclusión constituye la sección 4 final.

\section{El anti-psicologismo como teoría filosófica determinante de la naturaleza de la lógica}

En términos básicos el psicologismo es la doctrina según la cual las leyes de la lógica son reductibles a leyes psicológicas. R. Hanna, en su análisis sobre Husserl, lo ha expresado actualmente con una forma modal, según la cual: $\square \forall x$ (x es una proposición lógica $\rightarrow \exists y$ (y es humano e y piensa $\mathrm{x}$ )), donde $\mathrm{x}$ es una entidad mental. Lo que se dice es: "Necesariamente todas las proposiciones lógicas son ítems dentro de una particular mente humana" (Hanna 1993, p. 255). Ello también implica que las leyes de la lógica son en verdad leyes contingentes (Hanna 1993, p. 261). Por su parte el anti-psicologismo se expresa, en su sentido fuerte como: $\square \forall \mathrm{x}$ (x es una proposición lógica $\rightarrow \neg \exists$ y (y es un ser humano e y piensa en $x$ )), lo que afirma que necesariamente ninguna proposición lógica es un item mental humano. La versión débil del antipsicologismo cambia los operadores modales: $\diamond \forall x$ (x es una proposición lógica $\wedge \neg \exists y$ (y es humano e y piensa en $\mathrm{x}$ )), lo que afirma que posiblemente las proposiciones de la lógica no sean ítems mentales humanos (Hanna 1993, p.258). Cabe decir al respecto que otra postura antipsicologista común sería: no es un problema aceptar que una ley lógica sea un ítem mental, pues todo enunciado, sobre cualquier materia, lo sería, y ello es irrelevante para la lógica en cuanto disciplina. Tal vez sea esta la reacción más común entre los antipsicologistas más duros.

La obra de Frege es el locus clásico del anti-psicologismo. Afirma Frege, en una carta a Husserl de octubre de 1906:

Me parece que los lógicos se abrazan demasiado al lenguaje y a la gramática y están demasiado enmarañados en la psicología. (...) Encuentran que mi conceptografía no representa correctamente los procesos mentales; y tienen razón, pues éste no es en absoluto su propósito. (...) Parece que aún se considera como tarea de la lógica el estudiar procesos mentales. Realmente la lógica no tiene mucho más que ver con ellos que con el movimiento de los cuerpos celestes. No es en ningún sentido parte de la psicología (Frege 1998, p. 189).

En otro texto pertinente, Frege se hace cargo de una expresión muy utilizada por los lógicos, incluso desde la obra de Arnauld y Nicole, según la cual la lógica versaría sobre las "leyes del pensamiento". Frege advierte que allí se esconde un peligro, el peligro del psicologismo:

La expresión "ley del pensamiento" se entiende, quizás, por analogía con "ley de la naturaleza", queriendo hacer referencia mediante ella a lo general de los acontecimientos mentales del pensar.Una ley del pensamiento sería, en este sentido, 
una ley psicológica.Y así se podría llegar a creer que la lógica trata del proceso mental del pensar y de las leyes psicológicas de acuerdo con las cuales éste tiene lugar. Pero esto sería no comprender la tarea de la lógica puesto que la verdad no ocupa aquí el lugar que le corresponde (Frege 1998a, p. 223).

La tesis con la que Frege sustenta las ideas anteriores tiene que ver con la idea de "pensamiento", como diferente a la de "representación". El primero es objetivo, y a él pertenece la lógica, y el segundo es subjetivo, es el "mundo interior". La lógica no versa sobre represtaciones, que pertenecen a la psicología, sino solo sobre pensamientos. Dicho de una manera concentrada: Frege reconoce por cierto la existencia de un "mundo interior", subjetivo, lo que denomina "representación". Ahora bien, llama pensamientos no a las representaciones, que son algo que un sujeto en particular tiene o no tiene y que por ende le son propias e intransferibles, sino que a un tercer ámbito ontológico o mundo. Dos personas no pueden tener la misma representación: pueden tener, sí, cada uno su representación acerca del mismo objeto. Con el concepto de representación salva Frege el asunto de la subjetividad. Para Frege, el pensamiento no es una representación (Frege 1998, p. 211), pues es algo objetivo. El pensamiento expresado en el teorema de Thales es el mismo pensamiento para quienquiera que lo piense, pues de lo contrario habría tantos teoremas de Thales como sujetos lo piensen. La lógica pertenece al reino de la objetividad, no al mundo de los objetos del mundo exterior ni tampoco al mundo subjetivo de las representaciones. Esta es la idea central del anti-psicologismo.

Husserl, por su parte, también atacará al psicologismo y buscará una idea de una "lógica pura", más allá de toda relación con la experiencia. Para Husserl el psicologismo dominaba aquella etapa de la historia de la lógica ${ }^{5}$. Fundamentalmente la tesis del psicologismo según Husserl afirma que: "los fundamentos teoréticos esenciales de la lógica residen en la psicología (...) La lógica se relacionaría, pues, con la psicología como una rama de la tecnología química con la química o como la agrimensura con la geometría, etc.” (Husserl 2001, p. 67).

El aporte de Husserl a este asunto radica en que proporcionó una base para la idea de la normatividad de la lógica. Efectivamente, lo normativo se lo ha tomado, desde Kant, como una idea primitiva. Sin embargo, Husserl busca una fundamentación para tal idea y la encuentra y desarrolla en su idea de teoricidad. En general, las disciplinas normativas deben fundarse en las disciplinas teoréticas (Husserl 2001, pp. 64 y ss.).

De acuerdo con lo anterior, es defendible, tanto histórica como sistemáticamente, la idea de que en el debate entre psicologismo y anti-psicologismo no solo están involucradas dos visiones diferentes acerca de la lógica entre muchas posibles, sino

5 No cabe hacer aquí una historia del problema, pero, en síntesis, autores del siglo XIX fueron los defensores de la tesis psicologista no solo en lógica sino que en amplios sectores de la filosofía. Algunos de ellos fueron Wundt, Beneke, Sigwart, entre otros. Por otra parte, el anti-psicologismo tuvo como sus adalides a Bolzano y principalmente a Frege y Husserl, además de nombres como Pap, Feigl o Radnitzky. Pueden verse al respecto los artículos incluidos en D. Jacquette, ed., 2003, dedicado a una revisión actual del tema. 
que está en juego el concepto mismo de lógica. Esto es, la lógica se terminó de definir en su naturaleza última como antipsicologista; inversamente, afirmar el psicologismo implica la referencia a algo diferente a la lógica. Puede decirse que el núcleo del antipsicologismo consiste en afirmar que si la lógica ha de ser esencialmente simbólicoformal, la psicología no puede tener ninguna providencia en su naturaleza esencial. Es la idea de forma lógica la que resulta incompatible con la psicología; si la lógica es necesaria, a priori, simbólica, normativa, válida y neutral respecto del tópico, es gracias a que es formal; a que sus objetos, estrictamente, no son relaciones inferenciales entre enunciados sino que entre las formas de esos enunciados. Y la relación inferencial misma, a su vez, es formal. Y ello implica que el psicologismo esté radicalmente descaminado como filosofía de la lógica.

De allí, por ejemplo, que Peirce asoció la lógica con la simbolización, con la transformación de signos, esto es, con una semiótica. Y por ello recusó claramente el psicologismo. Las capacidades psicológicas, afirma Peirce, que pudieran estar implicadas en las transformaciones de símbolos al realizar una inferencia, no tienen ninguna relevancia para el hecho de que esa inferencia sea válida o no válida (Colapietro 2003, p. 165). De acuerdo con Colapietro, la idea de Peirce es que el psicologismo es imposible debido a que la forma lógica está dada ya en el signo mismo, sin necesidad de que una mente lo aprehenda, como afirma el psicologismo. Así, hay una forma lógica de esa inferencia, mas puede haber muchos pensamientos acerca de ella. El símbolo en cuanto tal es independiente de la mente y sus procesos. Y solo el símbolo es el dador de forma a la lógica. El símbolo revela la forma lógica (ibid, 165). Afirma Peirce: "La lógica, en su sentido general es, como creo haber mostrado, solamente otro nombre para la semiótica, la cuasi-necesaria o formal doctrina de los signos" (Peirce 1955, p. 98).

La cuestión del simbolismo conduce a considerar en este punto a la formalidad como elemento central de las concepciones de la filosofía de la lógica. La cuestión de la forma lógica ligada a un anti-psicologismo puede retrotraerse, en sus aspectos centrales, hasta Kant. La idea de una lógica pura, más allá de todas las condiciones empíricas y subjetivas, de los sentidos, imaginación, memoria, inclinaciones, prejuicios, etc., está ya en la Crítica de la Razón Pura. La Lógica Trascendental es una teoría acerca de esa idea, según la cual la lógica es un canon de la razón (más que un organon, como lo fue para Aristóteles). Por ello, la lógica es una disciplina de principios a priori (Kant B74, 2002, pp. 92 y ss.). El nudo de esta concepción no es sino aquel que mantiene unida a toda la Crítica: la idea de forma. Tal es la idea con la que Kant se habría quedado finalmente, no obstante que también consideró una lógica general aplicada, que versa sobre las reglas bajo condiciones empíricas, aunque sin atender a las diferencias entre objetos, por lo cual es general. Tal vez el dictum más representativo de lo que se entiende por lógica formal aún hoy está expresado en una reflexión de Kant en su Lógica: "No psicología: cómo se piensa, sino: cómo se debe pensar” (Kant 2000, Reflexión $\mathrm{N}^{\circ} 1.629$, p. 88).

En suma, si se analiza la caracterización de lo que se entiende por lógica se obtiene inmediatamente el debate sobre el psicologismo. Y, al revés, si se piensa en el debate sobre el psicologismo, se obtiene la figura de lo que ha sido pensado por lógica. Es notorio, por ejemplo, el recorrido que hace Jacquette al respecto al revisar los argumentos del anti-psicologismo y sus críticas. La lógica posee justamente las 
características que serían las contrarias a las de la psicología: exacitud, apriorismo, que conlleva necesidad, normatividad, universalidad, objetividad (Jacquette 2003, pp. 9 y ss.).

\section{Filosofía de la lógica y cognición: más allá del anti-psicologismo}

El resurgimiento actual de la cuestión psicologista tiene un signo muy distinto del debate original: no ha significado una vuelta al psicologismo y no se trata hoy, contra lo que piensa Dummett, de elegir entre psicologismo y no psicologismo como aquello que define la lógica. Significa, más bien, entender que la lógica consiste en algo más complejo que la mera formalidad sin contenido. La filosofía cognitiva de la lógica permite aunar formalidad simbólica con psicología cognitiva del razonamiento.

Tal vez una de las razones de por qué se ha producido el resurgimiento del debate sobre el psicologismo en la lógica radique en las concepciones sobre lo psicológico ayer y hoy. De acuerdo con lo expuesto en la sección anterior, Frege concebía la psicología, en cuanto ciencia, como aquel reino de la subjetividad, de las emociones, de las inclinaciones, esto es, de lo estrictamente propio de cada sujeto, intransferible y apenas comunicable. La introspección era el método privilegiado de acceso a esa interioridad, hacia las representaciones, esa región de lo ultrasubjetivo. En tal sentido, la idea de la objetividad de la lógica aparecía plenamente justificable en cuanto el ámbito opuesto e irreductible a lo psicológico. Pero, la psicología del razonamiento, y la psicología en general ha mutado desde lo inefable a la figura de cualquier ciencia fáctica, esto es, a la búsqueda de determinaciones regulares, de leyes, de hipótesis y teorías contrastables, que no solo describe sino que hipotetiza y elabora explicaciones ${ }^{6}$.

En lo que sigue, se examinarán dos ámbitos de investigación en la ciencia cognitiva que proporcionan antecedentes que apoyan una superación del debate psicologismoanti-psicologismo, a saber:

I. La forma lógica y los dominios cognitivos

II. Las facultades cognitivas y la lógica.

6 Las teorías cognitivas del razonamiento son hoy muy diversas. Van desde los enfoques de la lógica mental (Rips 2008) hasta la tesis de los razonamientos basados en modelos o las actuales formalizaciones computacionales de los razonamientos default (Carnota 1995), la abducción en I.A (Maganani 2001, Kowalski 2010), los enfoques probabilistas, los diversos approaches integrativos, los enfoques duales S1-S2 (Oaksford y Chater 2010), las teorías heurísticas (Tversky y Kahneman 1982). Para una taxonomía de los diferentes approaches actuales de Psicología del razonamiento ver Carretero et al. 1995. En relación con la confusión entre la subjetividad de las experiencias psicológicas y la psicología en cuanto ciencia empírica, confusión implicada en la propuesta de Frege, véase Jacquette 2003, p. 16. 


\section{La forma lógica y los dominios cognitivos}

Los dominios parecen complementar a la formalidad en la definición de la lógica. La contextualidad, aquí, posee muchas caras. Un primer tipo de contexto que puede mencionarse es el que rescata W. Stelzner (2003) respecto de la obra de Wundt. Wundt fue un psicologista, uno de los principales, fundador de la psicología moderna a partir de estudios fisiológicos. Pero, también, fue un lógico clásico que, desde el psicologismo, puso algunas bases para la actual lógica no clásica (esto mismo ha llevado a pensar a algunos autores que Wundt en realidad no es un buen representante del psicologismo después de todo). El significado de ello es que el psicologismo no ha sido nunca realmente el enemigo de la lógica formal simbólica, como pensaban Frege y Dummett. Afirma Stelzner: "Desarrollando y subrayando esos elementos no-clásicos, Wundt siguió su máxima según la cual la lógica no trata el conocimiento y la ciencia desde fuera, como un extraño, sino que la lógica misma 'depende de lo específico de las ciencias', lo cual significa que la lógica es aplicada". Allí mismo cita a Wundt: "La lógica no puede proceder bajo la condición de que las formas del pensamiento deberían ser indiferentes a los contenidos del conocimiento" (Stelzner 2003, p. 100). Sin embargo, resalta el autor, Wundt mismo no aceptaba ser un psicologista, en el sentido de que las leyes lógicas son parte o son reductibles a las leyes psicológicas o, en sentido ontológico, a estados mentales. Wundt aceptaba, por ejemplo, por completo que la lógica es normativa y la psicología es descriptiva. Y allí radica precisamente el interés sobre este autor: que establece una distinción a menudo desapercibida entre reducción y dependencia. Para Wundt, psicologismo no significa reducción de la lógica a la psicología; significa que no puede establecerse una completa independencia entre ambas.

En este ámbito, Stelzner resalta que Wundt, aún desde su especial psicologismo, estableció elementos para la lógica no clásica, específicamente acerca del concepto de negación. El concepto de negación, según Wundt, es contextual ${ }^{7}$. La negación de un concepto está en función de un conjunto de conceptos al cual pertenece el concepto negado. Así, cada negación contiene dos elementos: el concepto negado y el conjunto al cual pertenece ese concepto, su contexto. Esto da origen a una noción no clásica de negación que es ejemplificado así (Stelzner 2003, p. 105):

(1) Supongamos un conjunto $\mathrm{C}$ total de conceptos: $\mathrm{C}=\{\mathrm{a}, \mathrm{b}, \mathrm{c}\}$

(2) Entonces: $\neg \mathrm{a}$ es b o es c

$\neg$ b es a o es c

$\neg \mathrm{c}$ es a o es b

(3) Por lo que: $\neg \neg \mathrm{a}$ es $\neg \mathrm{b}$ o $\neg \mathrm{c}$

(4) Por tanto: $\neg \neg$ a es a o c; o a o b de (2) y (3)

(5) Entonces: $\neg \neg$ a es a o es b o es c de (4)

(6) De donde se puede decir que: $\neg \neg \mathrm{a} \neq \mathrm{a}$, dado $\mathrm{C}$

Posee un significado que coincide con el concepto pragmático de explicación post hempeliana. Ver van Fraassen (1990), W. Salmon (1974). 
C representa el contexto en el cual se da la negación del concepto. La negación de a, por ejemplo, lo es respecto de b y c. Ese contexto puede ser, y así es en el caso de Wundt, el contexto del conocimiento científico. Este enfoque permitió a Wundt pensar en la negación en términos no clásicos: así, según el ejemplo anterior, la doble negación clásica no es válida en esta concepción contextual; "no no a" no equivale, pues, a "a", sino que a algo más incierto, pues equivale sea a "a", pero también a "b" y a "c", en posibilidades abiertas ${ }^{8}$. Se puede seguir de aquí que la lógica no responde solo a la pura forma sino que es sensible al contexto. Pero al contexto se lo puede interpretar aquí como la presencia del sujeto; por ejemplo, el sujeto epistémico, en cuanto el contexto es un conjunto de conceptos científicos. El psicologismo de Wundt, apunta Stelzner, contiene un aporte a la lógica formal simbólica ${ }^{9}$. Es relevante señalar que ese contextualismo es perfectamente compatible con un tratamiento formal simbólico del razonamiento. Y ello es lo que normalmente ha venido haciendo la lógica no clásica, o las llamadas (extrañamente) "lógicas filosóficas". Se colige que este contextualismo cognitivo da un apoyo a teorías lógicas específicas, en este caso, a un sistema con negación no clásica.

Muchas aproximaciones en ciencias cognitivas pueden interpretarse como enfoques contextuales sobre la naturaleza de la lógica. Un caso de ello es la tesis de la interpretación y del dominio, de Stennings y van Lambalgen. Los autores afirman que la ciencia cognitiva está llevando a "[r]epensar el sentido en el cual la lógica puede ser considerada normativa” (2008, p. 11). Y agregan:

Las normas se aplican a instancias del razonamiento solamente después que la interpretación de las expresiones (lógicas y no lógicas) en el argumento han sido fijadas y que, además, hay en general múltiples opciones naturales para cada interpretación, aún para la interpretación de las expresiones lógicas (Stennings y van Lambalgen 2008, p. 11).

La tesis de los autores es que el razonamiento en general es simultáneamente formal y relativo al dominio. El punto relevante es la simultaneidad, porque nada impediría, si no fuese así, que se dijera que la lógica se encarga de la forma y la psicología del dominio. Mas, esta segunda alternativa no es sino la postura clásica del anti-psicologismo, que los autores niegan mediante el recurso al entrelazamiento de los dos factores. Esto quiere decir que la naturaleza misma de la lógica ya no depende del concepto de forma solamente. Esta postura se inscribe en una tesis más amplia sustentada por los autores: la lógica formal y la psicología cognitiva deben caminar juntas. La lógica, específicamente, es relevante para la psicología del razonamiento, postura, por lo demás, no comúnmente afirmada por los investigadores del razonamiento en ciencia cognitiva. Así, no cabe, en esta perspectiva, un anti-psicologismo como esencia de la lógica.

8 En esto, la tesis de Wundt está cerca del intuicionismo, que acepta como verdadero: $\mathrm{p} \rightarrow \neg\urcorner \mathrm{p}$, pero no que sea verdadero: $\neg \neg \mathrm{p} \rightarrow \mathrm{p}$.

9 Stelzner (2003) también analiza otro caso histórico en el que un lógico psicologista, como C.Sigwart, siglo 19, influyó en los desarrollos de las lógicas modales. 
La preeminencia tradicional de la pura forma en la comprensión de la lógica, según los autores, se hace patente en la manera en que se indaga si un razonamiento en particular es válido: primero, se lo traslada a la formalidad de la lógica del sistema adecuado y, luego, se lo compara con los patrones formales de validez de la lógica. La validez lógica reside en la forma de la regla; como tal, en expresión de Ryle, es neutral respecto del tópico. Supone que cualquier cosa que se reemplace (sustitución uniforme) por las constantes no lógicas de una expresión, su verdad se mantiene, y, en el caso de una inferencia, se mantiene su validez. Así, la validez puede ser comprobada por cualquiera y, sobre todo, en cualquier circunstancia. Esta concepción estándar de la validez / verdad no parece suficiente.

Los autores afirman que la lógica no es independiente del dominio, lo que es otra forma de afirmar que es contextual. Un esquema es válido dependiendo del dominio sobre el cual se razona con un propósito determinado. El razonamiento consiste en establecer primero el dominio sobre el que se razona y sus propiedades formales; solo después de ello el razonamiento puede ser guiado por leyes formales. Mas, ¿de qué cosas consta el dominio? Acciones, planes, contratos, normas, creencias, objetos matemáticos, leyes naturales, cosas, etc. (Stennings y van Lambalgen 2008, p. 24). Formalmente, un dominio es caracterizado por un conjunto de representaciones matemáticas, de estructuras, junto con un lenguaje formal. Luego, una relación de satisfacción, esto es, cómo dicho lenguaje es interpretado sobre el conjunto relevante de estructuras. Por ejemplo, la lógica proposicional clásica se aplica al dominio D de la matemática, es contextual a ese dominio; pero no lo es respecto del dominio D' de los razonamientos de sentido común. La lógica trivalente representa, en este enfoque, la extensión de un predicado al cual las cosas pertenecen en un grado (y, lo mismo, pero más radicalmente, para la lógica fuzzy); no sirve para otro dominio. Es el caso, por ejemplo, cuando las personas clasifican, afirman los autores, las cosas por matices, gradualmente (conjunto fuzzy), no en "pertenece" o "no pertenece" (conjunto crisp).

Los autores desarrollan largamente su tesis a partir del análisis del experimento cognitivo del razonamiento de selección, de Wason, canónico ya, a estas alturas ${ }^{10}$. Wason supuso que las respuestas de los sujetos del experimento modelarían el razonamiento sobre la base de que la regla condicional tiene una sola forma lógica, que tampoco se deja expresar en lógica proposicional sino que conviene a la lógica de primer orden. Los autores ofrecen la siguiente expresión formal de la regla en cuestión: sean los predicados: $\mathrm{V}(\mathrm{x}, \mathrm{y})=$ "x está en la cara visible de la carta $\mathrm{y}$ "; $\mathrm{I}(\mathrm{x}, \mathrm{y})=$ "x está en el lado

10 Puede verse dicho desarrollo de la tarea de selección, la madre de todas las tareas de razonamiento, como afirman los autores, en Stennings y Van Lambalgen, 2008, capítulo 3 , páginas 44 y siguientes. Pero, en lo esencial, la tarea de Wason consiste en presentar a un sujeto cuatro cartas, cada una con los signos A, K, 4, 7, respectivamente y la regla: "Si una carta tiene una vocal en un lado, tiene un número par en el otro". Se pregunta qué cartas de las cuatro se debieran voltear para confirmar o refutar la regla. Buena parte de la psicología del razonamiento actual se ha centrado en el análisis de esto. 
invisible"; $\mathrm{O}(\mathrm{x})=$ "x es una vocal"; $\mathrm{E}(\mathrm{x})$ = "x es un número par". Entonces, la regla, en una de sus alternativas, es:

\section{Rw: $\forall \mathrm{c}[\exists \mathrm{x}(\mathrm{V}(\mathrm{x}, \mathrm{c}) \wedge \mathrm{O}(\mathrm{x})) \rightarrow \exists \mathrm{y}(\mathrm{I}(\mathrm{y}, \mathrm{c}) \wedge \mathrm{E}(\mathrm{y}))]$ (Stennings y van Lambalgen 2008, pp. 53).}

Se asumió que esta es una expresión de la forma del condicional expresado en la regla, $\mathrm{p} \rightarrow \mathrm{q}$. Esto condujo a pensar que la lógica no podía dar cuenta de otros casos, no abstractos, en que se hacía intervenir el contenido, como por ejemplo: "Si tiene menos de 18 años entonces no puede beber". Los sujetos respondían mejor a este ejemplo que al abstracto de las 4 cartas, por lo que se ha pensado que la lógica no podía dar cuenta de los razonamientos reales. Pero, los autores plantean la tesis según la cual no es la lógica la que falla; más bien no se ha entendido la naturaleza de la lógica. La lógica tiene, diríamos, una naturaleza cognitiva, que va más allá de una forma lógica estándar. Pues lo que ocurre, como ya lo enunciamos anteriormente, es que la forma lógica es tributaria de una interpretación de un sujeto cognitivo ante un cierto problema. Ocurre que el condicional Rw tendrá la forma F1 para la interpretación cognitiva I1 y Fn para In. Así, hay al menos 3 interpretaciones para Rw, a) descriptiva, b) una deóntica y una tercera c) sobre la excepcionalidad. La primera interpretación, descriptiva del condicional, que es la única de ordinario asumida: $\mathrm{p} \rightarrow \mathrm{q}$, la que no da cuenta del ejemplo "si quiere beber debe tener 18 años o más". Incluso dentro de esta primera interpretación puede un sujeto entender la regla como: "un plan para decidir la verdad o falsedad de la regla"; o, también, "qué información se requiere para decidir la regla" o, aún "cómo obtengo dicha información". La segunda, b) es la deóntica, que conduce a otras formas lógicas: la regla ahora es: "debe ser el caso de que si hay una vocal en un lado debe haber un par en el otro": $\mathrm{O}(\mathrm{p} \rightarrow \mathrm{q})$, lo que nos pone en otro ámbito lógico; ahora el asunto es encontrar el conjunto que posiblemente viole la regla. Finalmente, la tercera interpretación del condicional lo relaciona con las excepciones a la regla. La tarea es vista ahora como la búsqueda de excepciones a la regla: la regla se aplica solo a cartas no excepcionales (Stennings y van Lambalgen 2008, p. 58), y su forma ahora es: $\mathrm{p} \wedge \neg \mathrm{e} \rightarrow \mathrm{q}$. Se trata de una tarea default, en que la regla se cumple si no se dan excepciones en contrario. Lo que el sujeto busca ahora es justamente esas excepciones que invalidarían la regla. No hay, en suma, una sola manera de afrontar la tarea de selección, lo que podría conducir a respuestas que pueden ser interpretadas como incorrectas en un dominio y correctas en otro. Las diversas interpretaciones nos ponen en diferentes dominios cognitivos.

La idea de forma lógica no puede desaparecer del concepto de la lógica. Pero sí puede ser transformada en el sentido de que está en función de algo anterior: que el enunciado (o regla de inferencia) debe ser previamente interpretado por un sujeto. Debe insistirse en que, en rigor, estos enfoques no implican que la lógica no sea simbolizable o que la forma no juegue un papel, incluso central. Pero la cuestión es que esa formalización y simbolización se conjuga con otros aspectos, en este caso, los contextos cognitivos del dominio. Podemos afirmar, según lo anterior, que la forma lógica es contextual. Podemos afirmar, también, que este enfoque interpretativo de la lógica implica algo más: implica que, al igual que las ideas de Wundt, da apoyo o justifica a las lógicas 
no clásicas, apoyo que se encuentra más allá de las puras consideraciones formales. En suma: si se puede expresar la forma general de un sistema lógico SL como: $S L=$ $<L, D>$ en su parte sintáctica (par lenguaje y base deductiva), al que se agrega una semántica $S$, deberemos agregar, también, un contexto o dominio $C$, en los términos de Stennings y van Lambalgen.

En este mismo sentido, acudiendo a las tesis de los autores, puede ser vista la cuestión de las llamadas lógicas de sentido común. En efecto, la Inteligencia artificial, I.A., ha dado cuenta de los tipos de razonamientos que no parecen ser del todo deductivos, con lo que se pone de relevancia la relación entre lógica y monotonía. En efecto, podemos entender la lógica default como una contextualización interpretativa epistémica respecto de la deducción. Si se quiere seguir aceptando a la lógica default como lógica de pleno derecho, debe aceptarse una idea débil de monotonía; si no se acepta tal debilitación, la lógica default debe quedar fuera de la lógica, más no de la formalización de los razonamientos.

La I.A. se ha interesado en representar este tipo de razonamientos. La cuestión básica es que la lógica clásica no se adecua a todas las formas de razonamientos en situaciones reales. Hay un tipo de razonamiento que es contextual al dominio epistemológico, esto es, a los conocimientos específicos con que cuenta un razonador en un momento determinado. "Si el bus parte a las 17 horas llega a las 21 horas"; tal enunciado, según la tesis del condicional material, si es verdadero, no puede permitir el caso de que el bus salga a las 17 y no llegue a las 21 . Sin embargo, en la realidad tal razonamiento depende de si el sujeto tiene un conocimiento adicional, "el bus sufre un desperfecto", de modo que el condicional, con esa nueva información, será verdadero, no obstante su antecedente es verdadero y su consecuente falso. La contextualidad aquí es obvia y prima por sobre la pura forma acontextual.

\section{Las facultades cognitivas y la lógica}

Pero no solamente los contextos son relevantes para el análisis de la forma en que es posible acoger factores cognitivos sin postular sea un psicologismo o sea un antipsicologismo. Ello se da también en la relación entre "facultades cognitivas" y su influencia en lo que se entiende por lógica. A. Goldman y el problema de la facultad de la percepción y R. Hanna, y su tesis de la facultad protológica, son autores que podemos relacionar con este tema.

El anti-psicologismo separa drásticamente lógica formal de facultad perceptiva y cualquier otro factor subjetivo. Si la lógica tiene carácter a priori, entonces no puede tener relación con la percepción, con la "experiencia". Sin embargo, A. Goldman retruca esta postura y muestra que ello es, en el mejor de los casos, dudoso. Aun cuando Goldman no lo trata, proponemos que, en su postura, está involucrada la perspectiva de la cognición distribuida (R. Giere 2002, 2004, A. Clark 2007, Hollan 2000). Afirma Goldman: "Notemos primero que en la práctica real los lógicos aprenden verdades leyendo las pruebas que otros han construido y construyendo sus propias pruebas con lápiz y papel. Esas actividades envuelven percepción” (Goldman 1986, p.301). 
Tradicionalmente se concebía que la cognición ocurre sin duda alguna en la mente y solo allí. Sin embargo, la ciencia cognitiva ha estado explorando la tesis revolucionaria de que en realidad la cognición es distribuida, esto es, no solo se daría en la mente sino que en otras instancias extra mentales. También, la distribución puede tener un carácter social, esto es, puede darse en distintos sujetos. Esta teoría cognitiva da un apoyo por lo pronto a las lógicas heterogéneas, a las ideas de la "superprueba" (Allwein y Barwise 1996), a la idea peirceana del carácter icónico fundamental de la lógica, en cuanto el ícono es representacional de alguna situación. La idea de que un diagrama, por ejemplo, no es un mero "apoyo" para una prueba sino que es el camino de una prueba propiamente tal, es una idea de distribución. En este sentido, la afirmación de Goldman, según la cual en una prueba participa, de derecho propio, una cierta instancia perceptual, no formal o simbólica, encuentra un fundamento en los desarrollos actuales en la teoría de la cognición distribuida en ciencia cognitiva.

Afirma Goldman, al respecto: "Uno podría, en principio, esquematizar todas las representaciones relevantes en la mente. Mas, ¿es eso así? ¿Capacitan las capacidades cognitivas humanas para construir, para seguir, una prueba compleja en la mente con suficiente confianza como para calificar como conocimiento? Es dudoso" (Goldman 1986, p. 302). Una de las razones es que la memoria de trabajo posee una capacidad limitada para tareas de pruebas demasiado complejas. Cuando la capacidad es excedida, sobreviene el error. Agrega: "En consecuencia, una verdad lógica cuya prueba envuelve este grado de complejidad puede ser sólo conocida con la ayuda de inscripciones externas, esto es, sólo con la ayuda de la percepción” (ibid, p. 302). Es notorio, pues, que aquello que Goldman denomina inscriptions corresponde a los elementos externos, extramentales, íconos peirceanos, diagramas, símbolos, gráficos, etc., que concurren a la prueba. En efecto, una ciencia como la óptica geométrica, ¿puede realmente ser pensada sin la representación gráfica? ¿Puede un arquitecto llegar a representar una idea sin el apoyo de la externalización perceptual? Las facultades perceptivas cognitivas son parte del razonamiento y, también, diríamos, de la lógica. Y la presencia de la percepción, como se puede colegir, no significa de ningún modo un retorno del psicologismo, esto es, del reduccionismo de la lógica a la "subjetividad".

Pero la percepción no es la única facultad cognitiva que pude concurrir a la comprensión de la naturaleza de la lógica. R. Hanna ha buscado una respuesta al fantasma del psicologismo apelando a las ideas de facultad lógica, protológica, animal lógico, lo que el autor denomina teoría del cognitivismo lógico. Dicha facultad protológica daría respuesta a la idea de que la naturaleza de la lógica no se define teniendo que tomar un partido entre psicologismo o anti-psicologismo, como cree Dummett. La tesis de Hanna puede encontrarse resumida en esta frase, de sabor kantiano:

la lógica es cognitivamente construida por los animales racionales, en el sentido de que sólo los animales racionales, incluidos los humanos, poseen una facultad cognitiva que es innata en su capacidad de representar la lógica, debido a que contiene una "protológica" universal, distinta en estructura de los sistemas lógicos clásicos y no clásicos, que es usada para construir todo sistema lógico. A esto lo llamo "la tesis de la facultad lógica (Hanna 2006, p. xiii). 
Y agrega, más adelante: “¿Pero, qué hay de la cuestión específica acerca de la naturaleza de la lógica? Mi respuesta es que la naturaleza de la lógica es explicada por la tesis de la facultad lógica: la lógica es cognitivamente construida por los animales racionales" (ibid, p. xv). La lógica es posible debido a la facultad humana de "logizar".

Las dos partes de la tesis, que la lógica es construida cognitivamente y que los seres racionales humanos poseen una facultad esencial, protológica, para representar lógicamente los sucesos, para "logizar", para estar "lógicamente orientado", se aúnan y ofrecen una imagen entrelazada entre lógica y cognición. Dicha unión es de muy larga historia, según el autor, que solo se vino a romper con la Conceptografía, de Frege. No hay espacio, pues, ni para el psicologismo ni para el anti-psicologismo. Es más, la naturaleza de la lógica, que de eso se trata finalmente, no puede ser conceptualizada desde el anti-psicologismo, como lo ha sido a partir del mito Frege-Husserl. El viejo mito aún está firme, afirma Hanna (1993, p. xiii). Solo una filosofía cognitiva de la lógica parece ser hoy el camino para romper, u olvidarse, del mito de la naturaleza antipsicológica de la lógica.

Hanna establece su posición en relación con las posturas de Chomsky y Fodor acerca de lo que es la razón. Ambos autores establecen una relación estrecha entre racionalidad y la capacidad cognitiva del lenguaje. Hanna rechaza la idea de que esa relación sea esencial (necesaria y suficiente). No es la facultad cognitiva para el lenguaje natural lo que define la razón; es la facultad cognitiva de la protológica. Esta facultad es la que permite afirmar que la lógica posee una base cognitiva, pero que ello no implica la postura filosófica del psicologismo.

El fundamento de la tesis de la facultad protológica el autor lo encuentra en un refinamiento del modelo estándar de la mente, cuyos parámetros son: representacionalismo, innatismo, constructivismo, modularidad y lenguaje mental. Nos detendremos solamente en la modularidad de la mente, por ser la más atingente al tema. La modularidad es una tesis acerca de cómo la mente está diseñada y estructurada. Muchos, quizás la mayoría, de nuestras capacidades cognitivas son especificas a un dominio y encapsuladas (Hanna 2006, ver pp. 88 y ss.). La modularidad posee hoy, según Hanna, amplia base evidencial. Por ejemplo, lo es el hecho de que una capacidad cognitiva puede deteriorarse (afasia, agnosia) sin que ello afecte otras capacidades. El caso del autista también es claro: la capacidad, digamos lingüística, o de simbolización no se pierde junto con su capacidad emocional.

Sin embargo, Hanna agrega una hipótesis a la teoría estándar de la mente modulada: y es que no toda capacidad cognitiva es encapsulada, esto es, independiente de otras. Hay al menos una capacidad cognitiva que es modular pero a la vez no encapsulada, lo que quiere decir que interactúa con otras capacidades de un sujeto cognitivo. Hay capacidades cognitivas que son "periféricas" esto es, que dan respuestas a inputs externos (sensoriales) y no a los provenientes de otras facultades. Por ello es encapsulada. En cambio, una facultad cognitiva central opera con las capacidades periféricas, en ambas direcciones, desde y hacia la periferia. Esta capacidad, por ende, no puede ser encapsulada, como lo afirma la teoría estándar. Y esta capacidad no encapsulada, no periférica, es 
la capacidad protológica. Ella tiene la misión de mediar entre los módulos periféricos y las teorías, juicios, creencias, deseos y voliciones; por ello no puede ser periférica.

La hipótesis es, entonces, que alguna capacidad debe hacer esa tarea mediadora; y esa es una capacidad de tipo lógico. Si es que es verdad la tesis de un lenguaje del pensamiento, que Hanna acepta, ella requiere una capacidad lógica básica del pensamiento. Pero lo interesante es que esa capacidad es protológica, dado que está en la base de la construcción de diversos sistemas lógicos, sean clásicos o no clásicos. La facultad protológica no responde a ningún sistema lógico en particular, al contrario de lo que afirmaba Piaget y su concepción de una "lógica mental". Más bien, la facultad protológica es condición cognitiva de posibilidad de cualquier sistema lógico construible. La capacidad mediadora no encapsulada es la capacidad de obedecer reglas, reflexionar sobre sí mismo, o tener conceptos lógicos como el de "consecuencia". Es inconcebible, afirma Hanna, que un ser racional lo sea sin tener una facultad protológica. Si la mente humana es esencialmente representacional, que incluye la representación a nivel sintáctico y semántico, la protológica es necesaria en la arquitectura de la mente.

\section{Conclusiones}

El anti-psicologismo se erigió como la norma de la filosofía de la lógica. Si un sistema es lógico entonces debe expulsar de sí toda relación con instancias psicológicas. Esa herencia fregeana hoy no pareciera sostenible completamente. Y, por ello, tampoco parecieran sostenerse del todo opiniones como las de M.Dummett, para quien hoy el peligro del psicologismo está de vuelta, ahora bajo la influencia de la ciencia cognitiva.

Al observar algunos desarrollos actuales de la ciencia cognitiva, especialmente de la Inteligencia artificial y la psicología del razonamiento, se puede concluir al menos lo siguiente: que los factores contextuales y las facultades cognitivas reguladas por la facultad protológica pueden conducir a la filosofía de la lógica a ampliar las concepciones que normalmente se sostienen acerca de la naturaleza de lo lógico. Y ello no implica de ningún modo un nuevo psicologismo, sino que la búsqueda de una concepción de la esencia de la lógica como algo más complejo, más allá de la pura forma simbólica.

\section{Referencias bibliográficas}

Aliseda A. (2006), Abductive Reasoning. Dordrecht: Springer.

(1998), "La abducción como cambio epistémico: C.S.Peirce y las teorías epistémicos en inteligencia artificial”, Analogía 12: 125-144.

Allwein G. y J. Barwise, eds. (1996), Logical Reasoning with Diagrams. Oxford / Nueva York: Oxford University Press.

Carnota R. (1995), “Lógica e inteligencia artificial”, en Alchourrón et al., eds., Lógica, EIAF, Madrid: Trotta; pp. 143-183.

Carretero M. et al. (1995), Razonamiento y comprensión. Madrid: Trotta. 
Clark A. (2007), "Curing Cognitive Hiccups: A Defense of the Extended Mind", The Journal of Philosophy 4: 163-192.

Colapietro V. (2003), “The Space of Sings: C.S. Peirce's Critique of Psychologism”, en Jacquette ed., Philosophy, Psychology, and Psychologism Critical and Historical Readings on the Psychological Turn in Philosophy.

Fernández P. y M. Carretero (1995), "Perspectivas actuales en el estudio del razonamiento", en Carretero et al., eds., Razonamiento y comprensión. Madrid: Trotta; pp.13-46.

Frege G. (1998), “Cartas a Husserl”, Frege, Ensayos de semántica y filosofía de la lógica. Madrid: Tecnos.

(1998a), "El pensamiento: una investigación lógica", en Frege, Ensayos de semántica y filosofía de la lógica. Madrid: Tecnos.

Giere R. (2002), "Models as Parts of Distributed Cognitive Systems”, en Magnani y Nersessian, eds., Model Based Reasoning: Science, Technology, values. Dordrecht: Kluwer, pp. 1-15.

(2004), "The Problem of Agency in Scientific Distributed Cognitive Systems", Journal of Cognition 4.3: 759-774.

Goldman A. (1986), Epistemology and Cognition. Cambridge: Harvard University Press.

Haack S. (1991), Filosofía de las lógicas. Madrid: Cátedra.

Hanna R. (1993), "Logical Cognition: Husserl's prolegomena and the Truth in Psychologism", Philosophy and Phenomenological Research $\mathrm{N}^{\circ}$ 53: 251-76. (2006), Rationality and Logic. Cambridge: MIT Press.

Hollan J., E. Hutchins y D. Kirsh (2000), “Distributed Cognition: A New Foundations for Human-Computer Interaction research, TOCHI vol 7, N²: 174-196.

Hjortland O. (2017), “Anti-exceptionalism about logic”, Philos Stud 174: 631-658.

Husserl E. (2001), Investigaciones lógicas I. Madrid: Alianza Editorial.

Jacquette D. (2003), "Introduction; Psychologism the Philosophical Shibboleth", en Jacquette ed., Philosophy, Psychology, and Psychologism Critical and Historical Readings on the Psychological Turn in Philosophy.

editor (2003), Philosophy, Psychology and Psychologism. Dordrecht: Kluwer.

Johnson-Laird P. (1995), Mental Models. Cambridge: Harvard University Press. Press. (1988), The Computer and the Mind. Cambridge: Harvard University (2005), "Mental Models and Thought", en Holyoak y Morrison eds., The Cambridge Handbook of Thinking and Reasoning. Cambridge, pp. 185-208.

(1991), "Mental Models", M. Posner ed., Foundations of Cognitive Science. Cambridge: MIT Press.

Kant I. (2002), Crítica de la razón pura. Madrid: Alfaguara. (2000), Lógica. Madrid: Akal. 
Kowalski R, (2010), "Reasoning with Conditionals in Artificial Intelligence”, en Mike Oaksford and N. Chater, eds., Cognition and Conditionals: Probability and Logic in Human Thinking. Oxford, UK: Oxford University Press, pp. 253-288.

Magnani L. (2001), Abduction, Reason and Science. Nueva York/ Dordrecht: Kluwer. McNamara J. (1994), "Logia and Cognition", en McNamara y Reyes, The Logical Foundations of Cognition. Oxford: Oxford University Press.

Mill J. S. (1917), Sistema de lógica. Madrid: Daniel Jorro.

Nersessian N. (2008), Creating Scientific Concepts. Massachusetts: MIT Press.

Oaksford y Chater, eds. (2010), "Cognitons and Conditonals". Oxford: Oxford University Press.

Peirce, Ch. S. (1955), "Logic as Semiotic", en Buchler J., ed., Philosophical Writings of Charles Peirce. Nueva York: Dover.

Rips L. (1994), The Psychology of Proof. Cambridge: Bradford Book, MIT Press.

(2008), "Logical Approaches to Human Deductive Reasoning”, en Adler y Rips, eds., Reasoning. Cambridge/ Nueva Yoprk: Cambridge University Press, pp. 187-205.

Salmon W. et al. (1974), La justificación de la inducción. Madrid: Alianza Editorial.

Stennings K. y M. van Lambalgen (2008), Human Reasoning and Cognitive Science. Cambridge: MIT Press.

Stelzner W. (2003), "Psychologism and Non-classical Approaches in Traditional Logic, en D. Jacquette, ed., Philosophy, Psychology, and Psychologism Critical and Historical Readings on the Psychological Turn in Philosophy.

Thagard P. (1993), Computational Philosophy of Science. Massachusetts: MIT Press.

Thomson V. (2010), "Towards a Metacognitive Dual Process Theory of Conditional Reasoning”, en Mike Oaksford and N. Chater, eds., Cognition and Conditionals: Probability and Logic in Human Thinking. Oxford, UK: Oxford University Press.

Tversky B. (2005), “Visuospatial Reasoning”, en K. Holyoak y R. Morrison, eds., The Cambridge Handbook of Thinking and Reasoning. Massachusetts: Cambridge University Press.

Tversky A. y Kahneman D, 1982, “Judgment Under Uncertainty: Heuristics and Biases", en Kahneman et al., Judgment Under Uncertainty. Cambridge: Cambridge University Press; pp. 3-20.

Van Fraassen B. (1990), The Scientific Image. Oxford/Nueva York: Clarendon Press.

Verschueren N. y W. Schaeken (2010), “A multi-Layered Dual-Process Approach to Condicional Reasonoing", en Oaksford y Chater, eds., Cognition and Conditionals: Probability and Logic in Human Thinking. Oxford, UK: Oxford University Press. 